\title{
Changes in the Spectrum of Kidney Diseases: An Analysis of 40,759 Biopsy-Proven Cases from 2003 to 2014 in China
}

\author{
Jin-Hua Hou Hui-Xian Zhu Min-Lin Zhou Wei-Bo Le Cai-Hong Zeng \\ Shao-Shan Liang Feng Xu Dan-Dan Liang Si-Jia Shao Ye Liu Zhi-Hong Liu \\ National Clinical Research Center of Kidney Diseases, Jinling Hospital, Nanjing University School of Medicine, \\ Nanjing, China
}

\section{Keywords}

Epidemiology · Renal biopsy · Spectrum of kidney disease · China

\begin{abstract}
Objectives: To evaluate the changing spectrum of kidney diseases over time in China using renal biopsy-proven cases. Methods: All patients over the age of 14 years who were diagnosed with a kidney disease by renal biopsy in the Renal Biopsy Registry of the National Clinical Research Center of Kidney Diseases in Jinling Hospital, Nanjing, from 2003 to 2014 were included. Results: In total, 40,759 cases of renal biopsy were analyzed. The mean age of the patients was $36.59 \pm 14.12$ years. $52.0 \%$ of the patients were male. Primary glomerulonephritis (PGN), secondary glomerulonephritis, tubulointerstitial disease, and hereditary renal diseases accounted for $67.1,26.4,2.9$, and $2.5 \%$, respectively. IgA nephropathy (IgAN), membranous nephropathy (MN), minimal change disease, and focal segmental glomerulosclerosis were the leading PGN diagnoses. The frequency of MN increased significantly $(p<0.001$ ) by doubling from 2003 to 2014. An analysis by age category indicated that the fre-
\end{abstract}

() 2017 S. Karger AG, Basel quency of MN increased significantly over time $(p<0.001)$ in all age categories and increased by more than 2 times in the 14-24 age category. Lupus nephritis (LN) and HenochSchönlein purpura nephritis (HSPN) decreased significantly ( $p<0.001)$, diabetic nephropathy (DN) increased nearly twice $(p<0.001)$, monoclonal immunoglobulin deposition disease (MIDD) tripled ( $p<0.001)$, and hypertensive nephropathy $(\mathrm{HT})(p<0.001)$ and renal amyloidosis (AMY) $(p<$ 0.05 ) showed an upward trend. An analysis by age category showed that hepatitis B-related nephritis has significantly decreased in the 14-24 age category $(p<0.001)$. Conclusion: PGN continued to be the predominant kidney disease in China with IgAN being the most common PGN. The frequency of $M N$ increased significantly, with a maximum increase in young adults. LN and HSPN decreased significantly, DN and MIDD increased significantly, and HT and AMY also showed an increasing trend. The kidney disease trends presented in this study serve as a reference point for patient care, disease prevention, and public health interventions.

(C) 2017 S. Karger AG, Basel

Jin-Hua Hou and Hui-Xian Zhu contributed equally to this article as co-first authors.

\section{KARGER}

E-Mail karger@karger.com

www.karger.com/kdd
Dr. Zhi-Hong Liu, MD

National Clinical Research Center of Kidney Diseases Jinling Hospital, Nanjing University School of Medicine No. 305 Zhongshan East Road, Nanjing 210016 (China)

E-Mail liuzhihong@nju.edu.cn 


\section{Introduction}

In 2010, a global burden of disease analysis showed that the predominant diseases in the Chinese population had changed from being communicable diseases to being noncommunicable diseases over the past 20 years and that these noncommunicable diseases had become a leading public health problem in China [1]. Chronic kidney disease (CKD) is one of the most significant of these noncommunicable diseases because its burden has become increasingly severe [2]. At present, there are over 100 million people with CKD in China [3], a prevalence that most likely will continue to increase due to the increasing incidence of hypertension [4], obesity [5], and type 2 diabetes mellitus $[6,7]$ and due to the increasing average age of the population [8]. Therefore, updated epidemiological studies evaluating the spectrum of kidney diseases that lead to $\mathrm{CKD}$ are needed for the purposes of improving patient care, disease prevention, and public health interventions.

We have previously reported on the spectrum of kidney diseases based on 13,519 renal biopsy-proven cases from 1979 to 2002 [9]. In this study, we aimed to evaluate the changing trends in the spectrum of kidney disease based on 40,759 biopsy-proven cases from 2003 to 2014 in China.

\section{Methods}

\section{Study Participants}

From January 1st, 2003, to December 31st, 2014, 45,238 cases had been included in the Renal Biopsy Registry of the National Clinical Research Center of Kidney Diseases in Jinling Hospital, Nanjing. We reviewed these cases and excluded patients below the age of 14 years, those with missing demographic data, and those who had transplanted kidney biopsies or donor kidney biopsies. Additionally, biopsies containing $<10$ glomeruli were excluded from the analysis. Finally, 40,759 cases of renal biopsy were included. The study was approved by local ethics committees. Informed consent was obtained for renal biopsy from each patient. The research was in compliance with the Declaration of Helsinki.

\section{Data Source}

Biopsy specimens were routinely processed using standard light, immunofluorescence, and electron microscopy procedures. Clinical pathological diagnoses of all cases had been established by a consensus among experienced nephropathologists and nephrologists. Referring to the Histological Classification Scheme of Glomerular Diseases issued by the World Health Organization in 1995, classifications were made as follows: (1) primary glomerulonephritis (PGN) included IgA nephropathy (IgAN), membranous nephropathy $(\mathrm{MN})$, minimal change disease (MCD), focal segmental glomerulosclerosis (FSGS), mesangial proliferative glomerulonephritis (MsPGN), membranoproliferative glomerulone- phritis type 1 (MPGN I), dense deposit disease (DDD), MPGN III, C3 glomerulonephritis (C3 GN), endocapillary proliferative glomerulonephritis, and others; (2) secondary glomerulonephritis (SGN) included lupus nephritis (LN), Henoch-Schönlein purpura nephritis (HSPN), diabetic nephropathy (DN), hypertensive nephropathy (HT), obesity-related glomerulonephropathy (ORG), vasculitic renal damage (VAS), renal amyloidosis (AMY), monoclonal immunoglobulin deposition disease (MIDD), hepatitis Brelated nephritis (HBVN), and others; (3) tubulointerstitial disease included acute interstitial nephritis (AIN), chronic interstitial nephritis, acute tubular necrosis, aristolochic acid nephropathy, and others; (4) hereditary renal diseases included thin basement membrane nephropathy, Alport syndrome, lipoprotein glomerulopathy, Fabry disease, and others; (5) unclassified cases included those with undetermined diagnoses or those who were unable to be classified.

If a patient had multiple biopsies and all had the same kidney disease diagnosis, only the first biopsy was used in this study. If a patient had multiple biopsies each with different diagnoses, all of the diagnoses were classified and retained within the analysis. If more than one kidney disease diagnosis was made from a single biopsy specimen, all of the diagnoses were classified and retained within the analysis. For example, a patient with DN and IgAN counted as both DN and IgAN separately.

Demographic data, including age, gender, and date of biopsy, were abstracted from either medical records or biopsy referral forms completed by the referring nephrologists.

Statistical Analyses

We categorized the data into three consecutive 4-year time intervals (2003-2006, 2007-2010, and 2011-2014) for tabular presentation and data analysis and into twelve consecutive 1-year time intervals for plotting.

Continuous variables were expressed as means \pm standard deviation or medians (interquartile ranges) and were compared using ANOVA or Kruskal-Wallis testing as appropriate. Categorical variables were expressed as frequencies (percentages) and compared using the $\chi^{2}$ or Fisher exact test as appropriate. Differences between periods were evaluated using the $\chi^{2}$ test for trend.

We used a generalized additive logistic model to estimate trends in the frequencies of the most common glomerulopathies with adjustments for gender and renal biopsy era. A two-sided $p$ value of $<0.05$ was considered statistically significant. SAS software (version 9.1; SAS Institute Inc.) was used for statistical analysis.

\section{Results}

\section{Study Participants}

A total of 40,759 cases of renal biopsy were analyzed in this study. From these, there were 916 repeat biopsies in 858 patients; 67 of these repeat biopsies had different diagnoses from the initial biopsies and were included in the analysis. There were 464 patients with two different kidney disease diagnoses, and we retained and classified all of the diagnoses in the analysis. Ultimately, the study included 40,759 cases with 41,290 diagnoses. Biopsy frequencies 


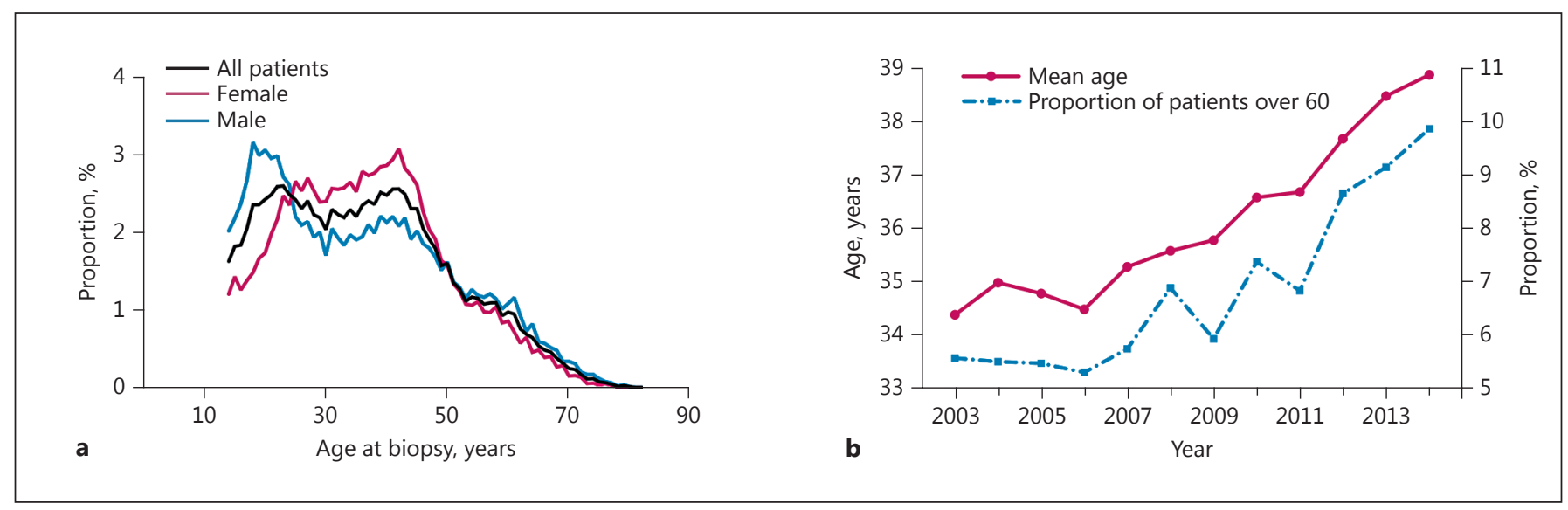

Fig. 1. Trends in age at biopsy. a Age distribution of renal biopsy patients. b Time-varying constituent ratio of mean age and renal biopsy patients aged over 60 years.

Table 1. Demographic characteristics of patients with renal biopsy

\begin{tabular}{lcccc}
\hline & $\begin{array}{l}2003-2006 \\
(n=8,447)\end{array}$ & $\begin{array}{l}2007-2010 \\
(n=14,078)\end{array}$ & $\begin{array}{l}2011-2014 \\
(n=18,234)\end{array}$ & $\begin{array}{l}\text { Total } \\
(n=40,759)\end{array}$ \\
\hline $\begin{array}{l}\text { Gender } \\
\quad \text { Male, } n(\%)\end{array}$ & $4,282(50.69)$ & $7,323(52.02)$ & $9,577(52.52)$ & $21,182(51.97)$ \\
$\quad \begin{array}{l}\text { Female, } n(\%) \\
\text { Age, mean } \pm \text { SD, years }\end{array}$ & $4,165(49.31)$ & $6,755(47.98)$ & $8,657(47.48)$ & $19,577(48.03)$ \\
Age category & $34.70 \pm 13.65$ & $35.94 \pm 14.01$ & $37.98 \pm 14.29$ & $36.59 \pm 14.12$ \\
$\quad \begin{array}{l}14-24 \text { years, } n(\%) \\
25-44 \text { years, } n(\%)\end{array}$ & $2,253(26.67)$ & $3,725(26.46)$ & $4,040(22.16)$ & $10,018(24.58)$ \\
$\quad 45-59$ years, $n(\%)$ & $1,371(16.23)$ & $2,822(20.05)$ & $4,537(24.88)$ & $8,730(21.42)$ \\
$\geq 60$ years, $n(\%)$ & $463(5.48)$ & $925(6.57)$ & $1,577(8.65)$ & $2,965(7.27)$ \\
\hline
\end{tabular}

increased annually from 1,978 (2003) to 4,861 (2014). Average patient age was $36.59( \pm 14.12)$ years (Table 1$)$. There were two distribution peaks in the age 20-25 and 40-45 categories of renal biopsy. A peak was observed among 20 -year-old males, followed by a plateau. Female cases were mainly concentrated in the age 25-45 category (Fig. 1a). Mean age of renal biopsy patients increased continuously. The percentage of patients aged $>60$ years increased from $5.48 \%$ in the period from 2003 to 2006 to $8.65 \%$ in the period from 2011 to 2014 ( $p<0.001$ ) (Fig. 1b). The ratio of male to female patients as a whole was 1:0.92 (male 52.0\%) (Table 1), which was almost equal to the data from the Chinese population census in 2010 (51.3\%).

\section{The Overall Changes in Renal Disease Frequencies}

PGN, SGN, tubulointerstitial disease, and hereditary renal diseases accounted for $67.07,26.42,2.94$, and $2.54 \%$, respectively, among cases of renal biopsy. PGN remains the most important and prevalent kidney disease in China, followed by SGN, tubulointerstitial disease, and hereditary renal diseases. Comparing the two periods of biopsy data in our renal center $(1979-2002$ [10] vs. $2003-2014)$, PGN (68.6\% vs. $67.07 \%, p<0.001)$ and tubulointerstitial disease ( 3.4 vs. $2.94 \%, p<0.001$ ) frequencies decreased between 1979-2002 and 2003-2014, while the frequencies of SGN $(24.5$ vs. $26.42 \%, p<0.001)$ and hereditary renal diseases ( 1.0 vs. $2.54 \%, p<0.001)$ increased significantly.

IgAN continued to be the most common PGN between both time intervals, and the second most prevalent PGN changed from mesangial proliferative glomerulonephritis to MN. Regarding SGN, the frequencies of LN and HSPN decreased, while the frequencies of DN, HT, and ORG increased (Fig. 2). No patients between 1979 and
12
Hou/Zhu/Zhou/Le/Zeng/Liang/Xu/Liang/ Shao/Liu/Liu 


\begin{tabular}{|c|c|c|c|c|c|c|}
\hline \multicolumn{3}{|r|}{ 1979-2002 } & \multicolumn{4}{|c|}{ 2003-2014 } \\
\hline Rank & Proportion & Primary glomerulonephritis & Primary glomerulonephritis & Proportion & Rank & Change \\
\hline 1 & $45.26 \%$ & IgA nephropathy & IgA nephropathy & $52.66 \%$ & 1 & $+7.40 \%$ \\
\hline \multirow{2}{*}{2} & \multirow{2}{*}{$25.62 \%$} & \multirow{2}{*}{$\begin{array}{l}\text { Mesangial proliferative } \\
\text { glomerulonephritis }\end{array}$} & Membranous nephropathy & $18.42 \%$ & 2 & $+8.53 \%$ \\
\hline & & & Minimal change disease & $14.81 \%$ & 3 & $+13.88 \%$ \\
\hline 3 & $9.89 \%$ & Membranous nephropathy & Focal segmental glomerulosclerosis & $7.34 \%$ & 4 & $+1.34 \%$ \\
\hline 4 & $6.00 \%$ & Focal segmental glomerulosclerosis & Mesangial proliferative & $2.74 \%$ & 5 & $-22.88 \%$ \\
\hline 5 & $3.38 \%$ & $\begin{array}{c}\text { Membranoproliferative } \\
\text { glomerulonephritis I + III }\end{array}$ & glomerulonephritis & & & \\
\hline 6 & $2.75 \%$ & $\begin{array}{l}\text { Endocapillary proliferative } \\
\text { glomerulonephritis }\end{array}$ & glomerulonephritis I + III & $1.30 \%$ & 6 & $-2.08 \%$ \\
\hline 7 & $0.93 \%$ & $\begin{array}{l}\text { glomerulonephritıs } \\
\text { Minimal change disease }\end{array}$ & $\begin{array}{c}\text { Endocapillary proliferative } \\
\text { glomerulonephritis }\end{array}$ & $0.73 \%$ & 7 & $-2.02 \%$ \\
\hline 8 & $0.03 \%$ & Dense deposit disease & C3 glomerulonephritis & $0.20 \%$ & 8 & $+0.20 \%$ \\
\hline 9 & 0 & C3 glomerulonephritis & Dense deposit disease & $0.05 \%$ & 9 & $+0.02 \%$ \\
\hline Rank & Proportion & Secondary glomerulonephritis & Secondary glomerulonephritis & Proportion & Rank & Change \\
\hline 1 & $54.68 \%$ & Lupus nephritis & Lupus nephritis & $32.93 \%$ & 1 & $-21.75 \%$ \\
\hline 2 & $25.62 \%$ & Henoch-Schönlein purpura nephritis & Diabetic nephropathy & $20.76 \%$ & 2 & $+14.11 \%$ \\
\hline 3 & $6,65 \%$ & Diabetic nephropathy & Henoch-Schönlein purpura nephritis & $17.04 \%$ & 3 & $-8.58 \%$ \\
\hline 4 & $4.11 \%$ & Vasculitic renal damage & Hypertensive nephropathy & $4.99 \%$ & 4 & $+3.13 \%$ \\
\hline 5 & $2.28 \%$ & Renal amyloidosis & Obesity-related & $4.94 \%$ & 5 & $+4.31 \%$ \\
\hline 6 & $1.86 \%$ & Hypertensive nephropathy & glomerulonephropathy & & & \\
\hline 7 & $1.05 \%$ & Monoclonal immunoglobulin & $\begin{array}{c}\text { Vasculitic renal damage } \\
\text { Renal amvloidosis }\end{array}$ & $\begin{array}{l}3.74 \% \\
3.63 \%\end{array}$ & $\frac{6}{7}$ & $-0.37 \%$ \\
\hline 8 & $1.02 \%$ & $\begin{array}{l}\text { deposition disease } \\
\text { Hepatitis B-related nephritis }\end{array}$ & Renal amyloıdosıs & $\begin{array}{l}3.63 \% \\
2.77 \%\end{array}$ & 8 & $\begin{array}{l}+1.35 \% \\
+1.75 \%\end{array}$ \\
\hline 9 & $0.63 \%$ & $\begin{array}{c}\text { Obesity-related } \\
\text { glomerulonephropathy }\end{array}$ & $\begin{array}{l}\text { Monoclonal immunoglobulin } \\
\text { deposition disease }\end{array}$ & $1.28 \%$ & 9 & $+0.23 \%$ \\
\hline & & 1979-2002 & $2003-2 C$ & & & \\
\hline Rank & Proportion & Tubulointerstitial disease & Tubulointerstitial disease & Proportion & Rank & Change \\
\hline 1 & $35.13 \%$ & Acute tubular necrosis & Acute interstitial nephritis & $45.21 \%$ & 1 & $+10.95 \%$ \\
\hline 2 & $34.26 \%$ & Acute interstitial nephritis & Acute tubular necrosis & $29.46 \%$ & 2 & $-5.67 \%$ \\
\hline 3 & $21.77 \%$ & Chronic interstitial nephritis & Chronic interstitial nephritis & $20.13 \%$ & 3 & $-1.64 \%$ \\
\hline 4 & $5.80 \%$ & Aristolochic acid nephropathy & Aristolochic acid nephropathy & $3.05 \%$ & 4 & $-2.75 \%$ \\
\hline 5 & $2.16 \%$ & Refux nephropathy & Bartter syndrome & $0.99 \%$ & 5 & $+0.13 \%$ \\
\hline 6 & $0.86 \%$ & Bartter syndrome & Refux nephropathy & $0.58 \%$ & 6 & $-1.58 \%$ \\
\hline 7 & 0 & Gitelman syndrome & Gitelman syndrome & $0.58 \%$ & 7 & $+0.58 \%$ \\
\hline Rank & Proportion & Hereditary renal disease & Hereditary renal disease & Proportion & Rank & Change \\
\hline 1 & $74.64 \%$ & Alport syndrome & Thin basement membrane & $48.43 \%$ & 1 & $+30.31 \%$ \\
\hline 2 & $18.12 \%$ & Thin basement membrane & nephropathy & & & \\
\hline 2 & $10.12 \%$ & nephropathy & Alport syndrome & $33.27 \%$ & 2 & $-41.37 \%$ \\
\hline 3 & $4.35 \%$ & Lipoprotein glomerulopathy & Lipoprotein glomerulopathy & $4.58 \%$ & 3 & $+0.23 \%$ \\
\hline 4 & $0.72 \%$ & Fabry disease & Fabry disease & $3.43 \%$ & 4 & $+2.71 \%$ \\
\hline
\end{tabular}

Fig. 2. Changes in renal pathology frequencies (1979-2002 vs. 2003-2014).

Changes in the Spectrum of Kidney Diseases in China 


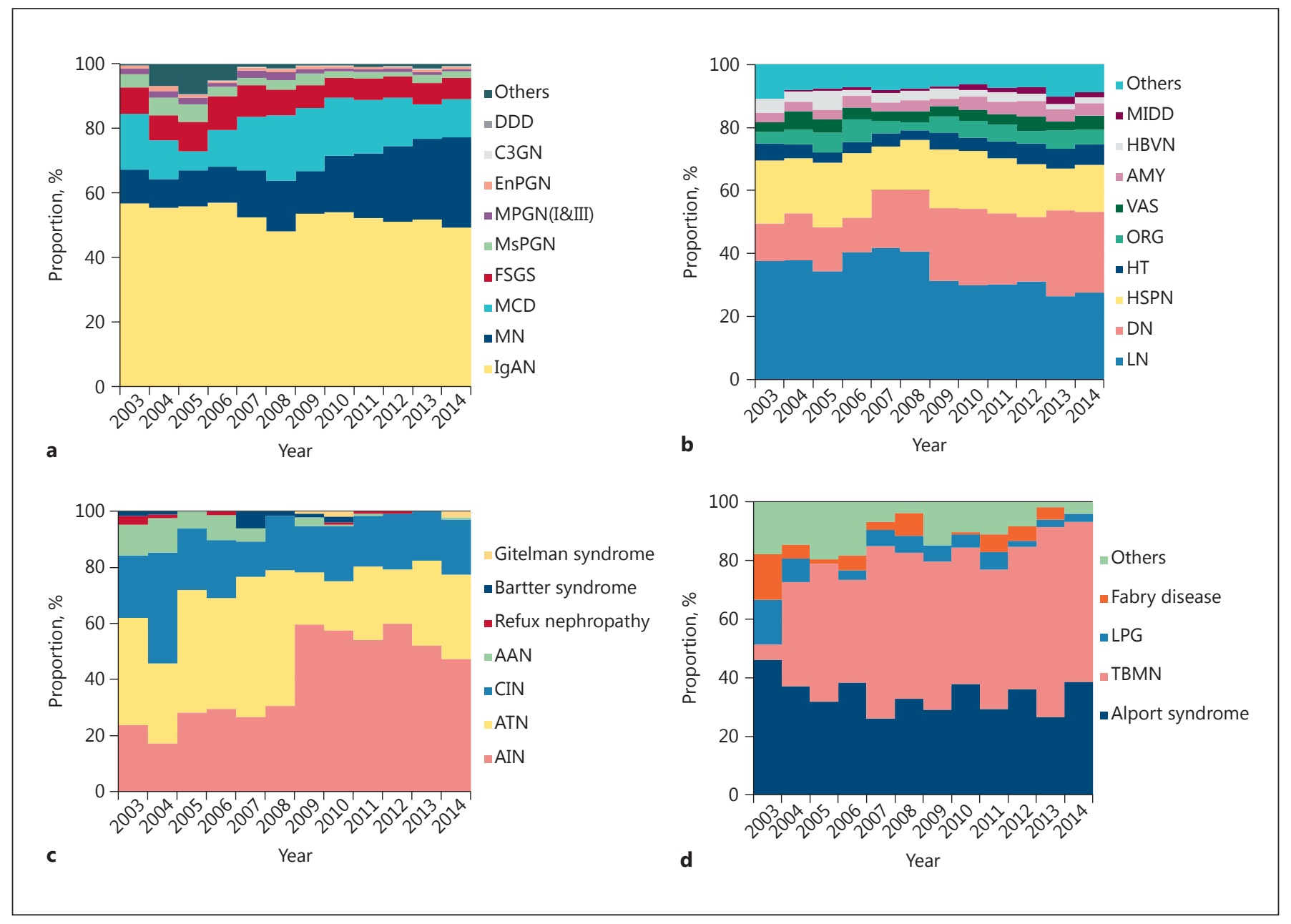

Fig. 3. Temporal trends of the renal biopsy frequencies of kidney diseases subtypes, 2003-2014. a Frequencies of primary glomerulonephritis subtypes. Percent of all primary glomerulonephritis categories. b Frequencies of secondary glomerulonephritis subtypes. Percent of all secondary glomerulonephritis categories. c Frequencies of tubulointerstitial disease subtypes. Percent of all tubulointerstitial disease categories. d Frequencies of hereditary renal disease subtypes. Percent of all hereditary renal disease categories. IgAN, IgA nephropathy; MCD, minimal change disease; $\mathrm{MN}$, membranous nephropathy; FSGS, focal segmental glomerulonephritis; MsPGN, mesangial proliferative glomerulonephritis; MPGN(I\&III), membranoproliferative glomerulonephritis type 1

2002 were diagnosed with rare kidney diseases, such as C3 glomerulonephritis and Gitelman syndrome. However, the frequencies of these kidney diseases have increased in the most recent decade.

\section{The Changes in PGN Frequencies}

IgAN, MN, MCD, and FSGS were the leading PGN diagnoses (Fig. 3a). IgAN occurred frequently in young and 3; EnPGN, endocapillary proliferative glomerulonephritis; C3GN, C3 glomerulonephritis; DDD, dense deposit disease; LN, lupus nephritis; DN, diabetic nephropathy; HSPN, HenochSchönlein purpura nephritis; HT, hypertensive nephropathy; ORG, obesity-related glomerulopathy; VAS, vasculitic renal damage; AMY, renal amyloidosis; HBVN, hepatitis B-related nephritis; MIDD, monoclonal immunoglobulin deposition disease; AIN, acute interstitial nephritis; ATN, acute tubular necrosis; CIN, chronic interstitial nephritis; AAN, aristolochic acid nephropathy; TBMN, thin basement membrane nephropathy; LPG, lipoprotein glomerulopathy.

adults, whereas the frequencies of MCD and FSGS decreased with age and tended to be stable after the age of 30. The constituent advantage of $\mathrm{MN}$ increased gradually and over half of patients over 60 had MN (Fig. 4a).

As shown in Table 2, the frequencies of IgAN and FSGS have decreased significantly $(p<0.001)$, while the frequency of MN increased significantly $(p<0.001)$ and nearly doubled from 2003-2006 to 2011-2014. The anal- 


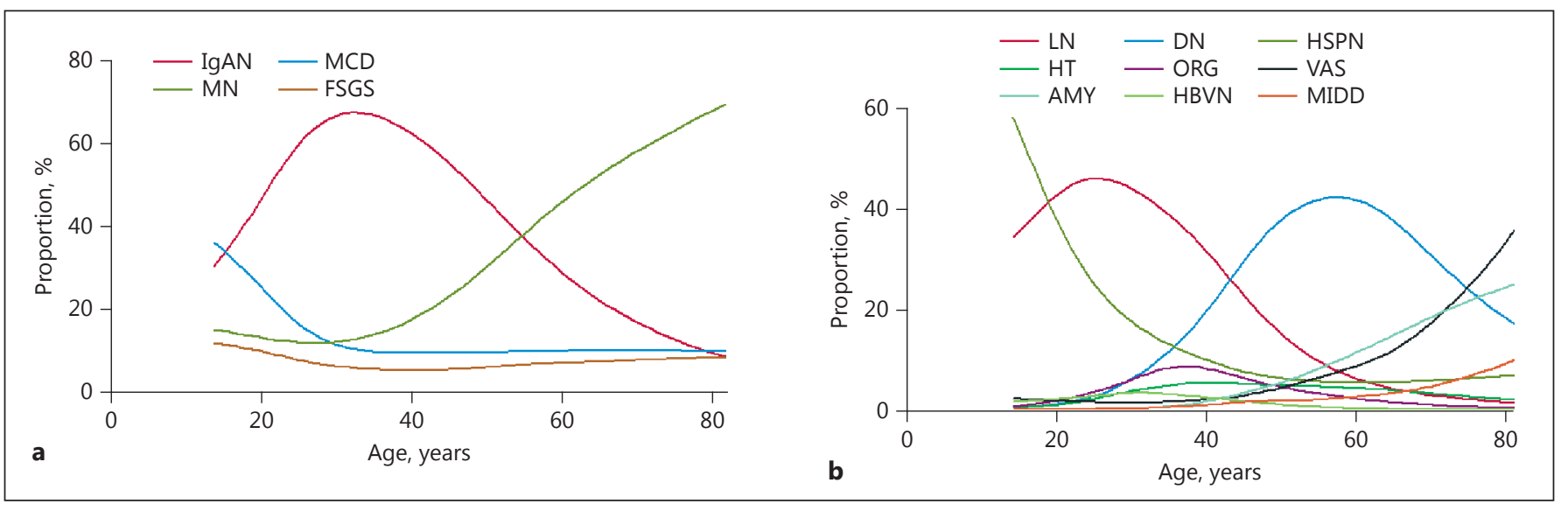

Fig. 4. Renal biopsy diagnosis frequencies of the most common glomerular disease subtypes according to patient age. The disease frequencies were estimated from generalized additive logistic models adjusted for gender and renal biopsy era. a The renal biopsy diagnosis frequencies of the most common primary glomerulonephritis subtypes. Percent of all primary glomerulonephritis categories. $\mathbf{b}$ The renal biopsy diagnosis frequencies of the most common secondary glomerulonephritis subtypes. Percent of all secondary glomerulonephritis categories.

ysis by age category indicated that the frequency of $\mathrm{MN}$ in all age categories increased significantly over time $(p<$ $0.001)$, and increased by more than 2 times in the 14-24 age category, which was the greatest increase in all age categories, while it minimally increased in the $\geq 60$ age category.

\section{The Changes in SGN Frequencies}

LN, HSPN, DN, HT, ORG, VAS, AMY, HBVN, and MIDD were the most common SGN (Fig. 3b) diagnoses. There were significant differences in the frequencies of each SGN in different age categories. LN peaked in the age $20-40$ category. HSPN peaked in the age $14-20$ category and decreased sharply in the succeeding age categories before stabilizing around age 50 and beyond. DN was most common in the age 45-70 category. The frequencies of AMY, VAS, and MIDD increased with age (Fig. 4b).

The constituent ratio of each SGN changed dynamically at different time intervals. LN and HSPN decreased significantly $(p<0.001)$, DN increased nearly twice $(p<$ $0.001)$, MIDD increased by 3 times $(p<0.001)$, and HT $(p<0.001)$ and AMY $(p<0.05)$ also increased and were especially prominent from 2011 to 2014 . The analysis by age category found that HBVN showed a significant downtrend in the $14-24$ age category only $(p<0.001)$ (Table 3).

\section{The Changes in Tubulointerstitial Disease and}

\section{Hereditary Renal Disease Frequencies}

The frequencies of tubulointerstitial disease are shown in Figure 3c. In total, there were 1,212 patients with tubulointerstitial disease. The most common tubulointerstitial disease was acute interstitial nephritis (45.21\%), followed by acute tubular necrosis $(29.46 \%)$, chronic interstitial nephritis (20.13\%), and aristolochic acid nephropathy (3.05\%).

The frequencies of hereditary renal diseases are shown in Figure 3d. There were 1,049 patients diagnosed with hereditary renal diseases. The most common hereditary renal diseases were Alport syndrome (33.27\%) and thin basement membrane disease (48.43\%). Lipoprotein glomerulopathy accounted for $4.58 \%$ of hereditary renal diseases. Fabry disease accounted for $3.43 \%$.

\section{Discussion}

This study analyzed and summarized patient characteristics and the changing frequencies of kidney diseases using the Renal Biopsy Registry of the National Clinical Research Center of Kidney Diseases in Jinling Hospital, Nanjing, from 2003 to 2014. The results indicated that in PGN the frequencies of IgAN and FSGS have decreased significantly, while the frequency of $\mathrm{MN}$ increased significantly, with a maximum increase in the 14-24 age category. In SGN, LN and HSPN decreased and HBVN de- 
Table 2. Temporal trends in the renal biopsy frequencies of the most common PGN subtypes by age strata

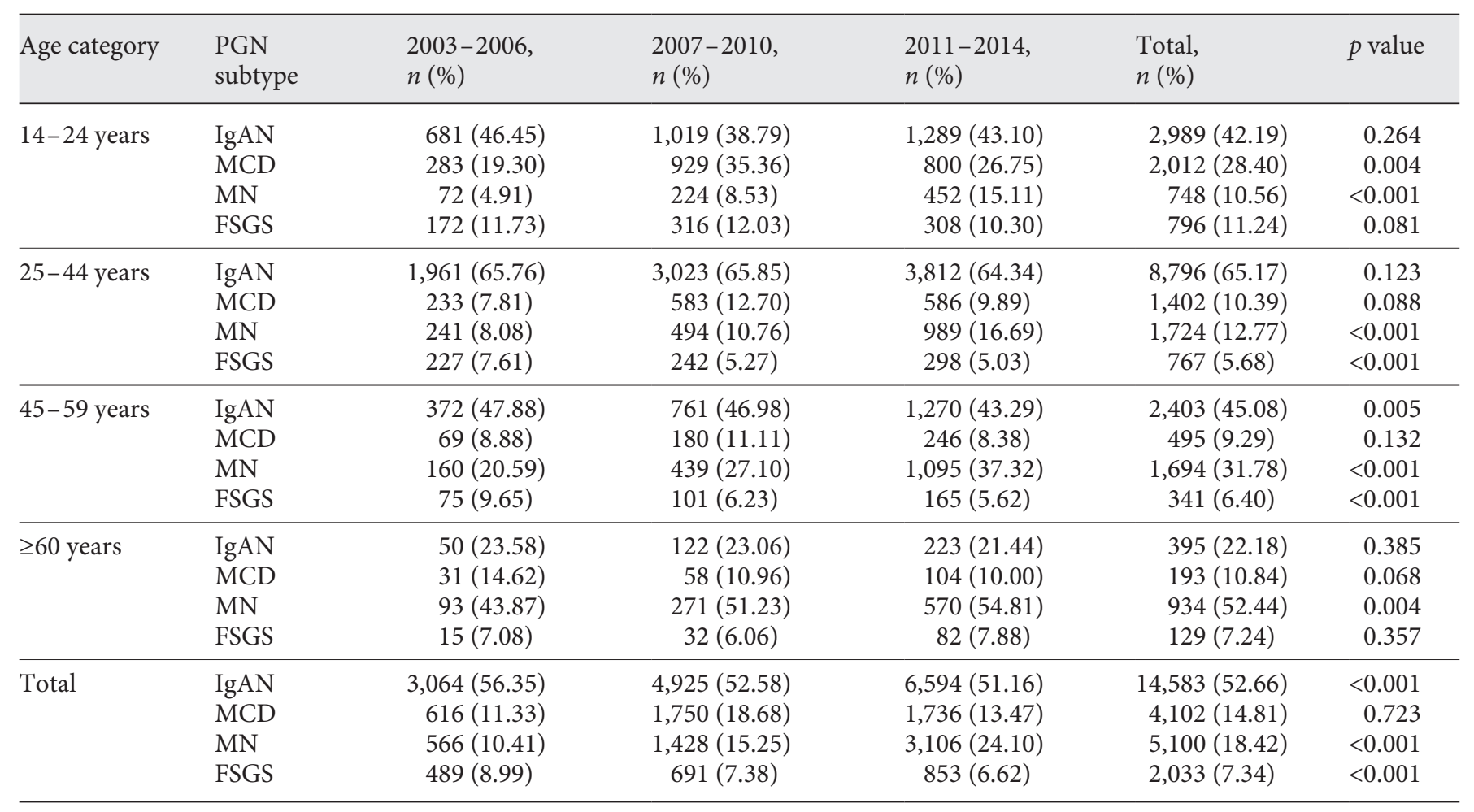

PGN, primary glomerulonephritis; IgAN, IgA nephropathy; MCD, minimal change disease; MN, membranous nephropathy; FSGS, focal segmental glomerulonephritis.

creased significantly in the 14-24 age category over time, whereas DN, HT, AMY, and MIDD increased over time.

The mean age of kidney disease patients has increased significantly in the past 12 years, and the frequency of patients over the age of 60 years with renal biopsy has increased. These trends are mostly likely due to the fact that renal biopsy has become more popular and because the technology has improved, meaning that age 60 years is no longer an absolute contraindication for renal biopsy. Additionally, the increasing average age of the Chinese population might also be one of the important reasons.

MN increased the most from 2003 to 2014 and has now become the second most common PGN. Previous studies [10-17] indicated that in China the majority of patients aged $>60$ years who underwent renal biopsy had MN, especially patients aged $\geq 65$ years. Studies have also shown that MN has increased significantly in India [18] and Brazil [19], while it decreased in Japan [20], Korea [21], the USA [22, 23], and the UK [24]. In addition to geographical and genetic factors, the increase in MN in China and the decrease in $\mathrm{MN}$ in developed countries might be related to environmental exposures, industrialization, and lifestyle changes [15]. Industrialization is inevitably accompanied by environmental pollution, involving heavy metals and organic solvents. A multicenter study in China reported that the frequency of $\mathrm{MN}$ presented a rising tendency and doubled from 2004 to 2014, which was consistent with our research results. Based on the analysis in that study, the high prevalence of MN was most likely associated with poor air quality, and suggested that longterm exposure to high levels of PM2.5 might be associated with an increased risk of MN [17]. However the specific mechanism remains to be further explored.

One of the important findings from our study is the remarkable rising trend in the frequency of $\mathrm{MN}$, especially in the age 14-24 category, which represented the greatest increase in all age categories, while it only minimally increased in the $\geq 60$ age category. Liu et al. [25] analyzed the spectrum of kidney disease in Chinese children (6-19 years old) and found that $\mathrm{MN}$ reached up to
16

Kidney Dis 2018;4:10-19 DOI: $10.1159 / 000484717$
Hou/Zhu/Zhou/Le/Zeng/Liang/Xu/Liang/ Shao/Liu/Liu 
Table 3. Temporal trends in the renal biopsy frequencies of the most common SGN subtypes by age strata

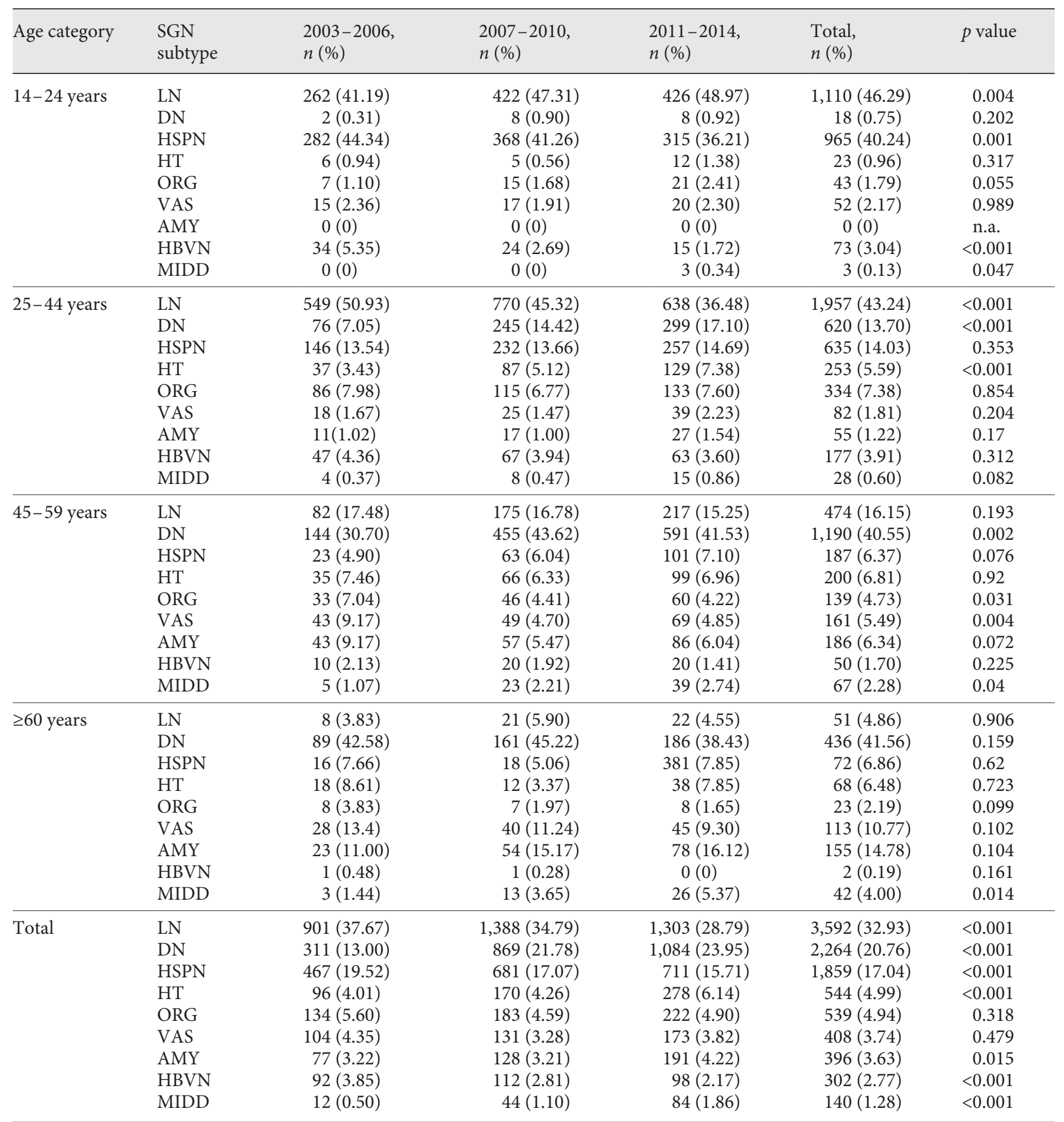

SGN, secondary glomerulonephritis; LN, lupus nephritis; DN, diabetic nephropathy; HSPN, Henoch-Schönlein purpura nephritis; HT, hypertensive nephropathy; ORG, obesity-related glomerulopathy; VAS, vasculitic renal damage; AMY, renal amyloidosis; HBVN, hepatitis B-related nephritis; MIDD, monoclonal immunoglobulin deposition disease; n.a., not available.

Changes in the Spectrum of Kidney

Diseases in China
Kidney Dis 2018;4:10-19 DOI: $10.1159 / 000484717$ 
$7.5 \%$ of all PGN, with a significant rising tendency from 2009 to 2013 (4.4 vs. 9.1\%). Therefore, the frequency of $\mathrm{MN}$ in young adults has increased, suggesting that the possibility of MN should be actively considered in young patients with nephrotic syndrome in clinical practice. The younger the patients were, the higher the rising frequency of $\mathrm{MN}$ was. The reason behind this phenomenon has yet to be elucidated. However, perhaps younger patients are more vulnerable to the effects of pollution and thus more susceptible to $\mathrm{MN}$.

The frequencies of some other PGN subtypes significantly declined, e.g. IgAN and FSGS, although IgAN is still the most common PGN. A similar finding was also observed in other Chinese subpopulations [17], while the frequency of IgAN has remained stable in the USA [23]. The decline in FSGS frequency has been previously observed in the Chinese population [17]. Previous studies from the USA showed an increase in the frequency of FSGS, which was followed by a plateau and decline in its frequency in the 21st century [23].

Renal damage secondary to diabetes and hypertension had a rising tendency. Considering renal biopsy indications, the patients considered as having DN or advanced $\mathrm{DN}$ in the clinic did not tend to receive renal biopsy, so the prevalence of DN might be underestimated, actually. As the prevalence of diabetes in the Chinese population increases continuously $[6,7], \mathrm{DN}$ will certainly maintain an increasing trend. Similarly in the USA, renal biopsyproven $\mathrm{DN}$ has markedly increased over the past 3 decades [23].

HT has also increased. A national epidemiological investigation [4] found that the prevalence of hypertension increased from $18.0 \%$ to $27.8 \%$ from 2002 to 2013 in China, which means that there were 139 million newly diagnosed patients with hypertension. Therefore, it is likely that HT will continue to increase.

The increase in the incidence of metabolic-related nephropathies suggests that the influence of metabolic factors should not be ignored when diagnosing kidney disease. Furthermore, patients with metabolic disorders should be educated regarding their risks of renal damage.

Our study found that HBVN decreased significantly in the age 14-24 category, most likely due to the ministry of health's introduction of the hepatitis B vaccine in 1992. Liang et al. [26] revealed that HBsAg seroprevalence reduced from 9.8 to $7.2 \%$ from 1992 to 2006 in China. Furthermore, infection risk was reduced in newborns and infants. It is believed that the frequency of HBVN in patients $>24$ years will decrease as vaccinated patients continue to age.
The frequencies of kidney diseases that historically had low incidences, such as MIDD and AMY, increased. Other kidney diseases, such as C3 glomerulonephritis and Gitelman syndrome, were only reported in the most recent decade. The underlying reasons for these increases may include an increase in the referral population size from 1,978 (2003) to 4,861 (2014), improvements in knowledge of these disease and in diagnostic techniques, or a true increases in disease incidence. A large multicenter study will be needed to identify the reasons.

Our study has several limitations. Firstly, as a singlecenter study, the enrolled patients may not have been an adequate representation of the entire Chinese population, although the patients did come from a variety of regions in China. It is also worth mentioning that this study only reflects the frequencies of kidney diseases among those who have undergone renal biopsy, and thus these findings may not be applicable to the general population. In addition, the National Clinical Research Center of Kidney Diseases may be more likely to identify rare diseases and thus overestimate their prevalence. Secondly, as it is an observational study, we could only observe the changes in the frequencies of kidney diseases and thus could not fully explain the reasons behind these changes. Hence, follow-up studies are required to elucidate the roles of various factors in the frequencies of kidney diseases.

In conclusion, PGN remained the predominant kidney disease type in China, of which IgAN was the most common. The frequency of MN increased significantly with the greatest increase in young adults. LN and HSPN decreased significantly, while DN and MIDD increased significantly, and HT and AMY showed an upward trend. The kidney disease trends presented in this study serve as a reference point for patient care, disease prevention, and public health interventions.

\section{Acknowledgments}

The authors thank Fei Gu (Medical Pfizer Investment Co. Ltd., Beijing, China) for her editorial assistance.

This study was supported by the National Key Technology R\&D Program (2015BAI12B05), the National Key Research and Development Program (2016YFC0904100), and the Ministry of Science and Technology of the People's Republic of China.

\section{Conflict of Interest Statement}

The authors have no conflicts of interest to declare.
18

Kidney Dis 2018;4:10-19 DOI: $10.1159 / 000484717$
Hou/Zhu/Zhou/Le/Zeng/Liang/Xu/Liang/ Shao/Liu/Liu 


\section{References}

1 Yang G, Wang Y, Zeng Y, Gao GF, Liang X, Zhou M, Wan X, Yu S, Jiang Y, Naghavi M, Vos T, Wang H, Lopez AD, Murray CJ: Rapid health transition in China, 1990-2010: findings from the global burden of disease study 2010. Lancet 2013;381:1987-2015.

2 Liu ZH: Nephrology in China. Nat Rev Nephrol 2013;9:523-528.

3 Zhang L, Wang F, Wang L, Wang W, Liu B, Liu J, Chen M, He Q, Liao Y, Yu X, Chen N, Zhang J-e, Hu Z, Liu F, Hong D, Ma L, Liu H, Zhou X, Chen J, Pan L, Chen W, Wang W, Li $\mathrm{X}$, Wang $\mathrm{H}$ : Prevalence of chronic kidney disease in China: a cross-sectional survey. Lancet 2012;379:815-822.

4 Li Y, Yang L, Wang L, Zhang M, Huang Z, Deng Q, Zhou M, Chen Z, Wang L: Burden of hypertension in China: a nationally representative survey of 174,621 adults. Int J Cardiol 2017;227:516-523.

5 Shen J, Goyal A, Sperling L: The emerging epidemic of obesity, diabetes, and the metabolic syndrome in China. Cardiol Res Pract 2012; 2012:178675.

6 Yang W, Lu J, Weng J, Jia W, Ji L, et al: Prevalence of diabetes among men and women in China. N Engl J Med 2010;362:1090-1101.

7 Xu Y, Wang L, He J, Bi Y, Li M, et al: Prevalence and control of diabetes in Chinese adults. JAMA 2013;310:948-959.

8 Feng Z, Liu C, Guan X, Mor V: China's rapidly aging population creates policy challenges in shaping a viable long-term care system. Health Aff (Millwood) 2012;31:2764-2773.

9 Li LS, Liu ZH: Epidemiologic data of renal diseases from a single unit in China: analysis based on 13,519 renal biopsies. Kidney Int 2004;66:920-923.

10 Jin B, Zeng C, Ge Y, Le W, Xie H, Chen H, Liang S, Xu F, Jiang S, Liu Z: The spectrum of biopsy-proven kidney diseases in elderly Chinese patients. Nephrol Dial Transplant 2014; 29:2251-2259.
11 Zhu P, Zhou FD, Zhao MH: The renal histopathology spectrum of elderly patients with kidney diseases: a study of 430 patients in a single Chinese center. Medicine (Baltimore) 2014;93:e226.

12 Chen Y, Li P, Cui C, Yuan A, Zhang K, Yu C: Biopsy-proven kidney diseases in the elderly: clinical characteristics, renal histopathological spectrum and prognostic factors. J Int Med Res 2016;44:1092-1102.

13 Pan X, Xu J, Ren H, Zhang W, Xu Y, Shen P, Li X, Wang W, Chen X, Wu P, Feng X, Hao C, Chen N: Changing spectrum of biopsyproven primary glomerular diseases over the past 15 years: a single-center study in China. Contrib Nephrol 2013;181:22-30.

14 Zhang X, Liu S, Tang L, Wu J, Chen P, Yin Z, Li M, Xie Y, Cai G, Wei R, Qiu Q, Wang Y, Shi $\mathrm{S}$, Chen X: Analysis of pathological data of renal biopsy at one single center in China from 1987 to 2012. Chin Med J (Engl) 2014;127: 1715-1720.

15 Zhu P, Zhou FD, Wang SX, Zhao MH, Wang HY: Increasing frequency of idiopathic membranous nephropathy in primary glomerular disease: a 10-year renal biopsy study from a single Chinese nephrology centre. Nephrology 2015;20:560-566.

16 Xu X, Ning Y, Shang W, Li M, Ku M, Li Q, Li Y, Dai W, Shao J, Zeng R, Han M, He X, Yao Y, Lv Y, Liu X, Ge S, Xu G: Analysis of 4,931 renal biopsy data in central China from 1994 to 2014. Ren Fail 2016;38:1021-1030.

17 Xu X, Wang G, Chen N, Lu T, Nie S, Xu G, Zhang $\mathrm{P}$, Luo Y, Wang Y, Wang X, Schwartz J, Geng J, Hou FF: Long-term exposure to air pollution and increased risk of membranous nephropathy in China. J Am Soc Nephrol 2016;27:3739-3746.

18 Narasimhan B, Chacko B, John GT, Korula A, Kirubakaran MG, Jacob CK: Characterization of kidney lesions in Indian adults: towards a renal biopsy registry. J Nephrol 2006;19:205210 .
19 Polito MG, de Moura LA, Kirsztajn GM: An overview on frequency of renal biopsy diagnosis in Brazil: clinical and pathological patterns based on 9,617 native kidney biopsies. Nephrol Dial Transplant 2010;25:490-496.

20 Sugiyama H, Yokoyama H, Sato H, Saito T, Kohda Y, et al: Japan renal biopsy registry: the first nationwide, web-based, and prospective registry system of renal biopsies in japan. Clin Exp Nephrol 2011;15:493-503.

21 Chang JH, Kim DK, Kim HW, Park SY, Yoo TH, Kim BS, Kang SW, Choi KH, Han DS, Jeong HJ, Lee HY: Changing prevalence of glomerular diseases in Korean adults: a review of 20 years of experience. Nephrol Dial Transplant 2009;24:2406-2410.

22 Braden GL, Mulhern JG, O'Shea MH, Nash SV, Ucci AA, Germain MJ: Changing incidence of glomerular diseases in adults. Am J Kidney Dis 2000;35:878-883.

23 O’Shaughnessy MM, Hogan SL, Poulton CJ Falk RJ, Singh HK, Nickeleit V, Jennette JC: Temporal and demographic trends in glomerular disease epidemiology in the southeastern United States, 1986-2015. Clin J Am Soc Nephrol 2017;12:614-623.

24 Hanko JB, Mullan RN, O'Rourke DM, McNamee PT, Maxwell AP, Courtney AE: The changing pattern of adult primary glomerular disease. Nephrol Dial Transplant 2009;24: 3050-3054.

25 Liu A, Wu H, Su Y, Wang L, Xu G: Idiopathic membranous nephropathy in children in China. Fetal Pediatr Pathol 2015;34:185-189.

26 Liang X, Bi S, Yang W, Wang L, Cui G, Cui F, Zhang Y, Liu J, Gong X, Chen Y, Wang F, Zheng H, Wang F, Guo J, Jia Z, Ma J, Wang H, Luo H, Li L, Jin S, Hadler SC, Wang Y: Epidemiological serosurvey of hepatitis $\mathrm{B}$ in China - declining HBV prevalence due to hepatitis B vaccination. Vaccine 2009;27: 6550-6557.
Changes in the Spectrum of Kidney

Diseases in China 